\section{O papel do complexo principal de histocompatibilidade na fisiologia da gravidez e na patogênese de complicações obstétricas}

\author{
Major histocompatibility complex: its role in \\ the physiology of pregnancy and in the \\ pathogenic mechanisms of obstetric \\ complications
}

\author{
Crésio Alves ${ }^{1}$ \\ Sâmia Veiga ${ }^{2}$ \\ Maria Betânia P. Toralles ${ }^{3}$ \\ Antônio Carlos Vieira Lopes 4 \\ 1-4 Hospital Universitário Professor Edgard Santos. Faculdade de \\ Medicina. Universidade Federal da Bahia. Rua Augusto Viana s.n, \\ Salvador, BA. Brasil. CEP: 40110-060. \\ E-mail: cresio.alves@uol.com.br
}

\begin{abstract}
The aim of this paper is to review Human Histocompatibility Antigens (HLA) structure and function, its detection methods, nomenclature and pathogenic mechanisms associated with pregnancy physiology and obstetrics diseases. Immunological equilibrium between mother and conceptus is indispensable for the maintenance of pregnancy. Molecules from the HLA - mainly HLA-G expressed in the mother-fetus interface - fulfill an important function in maternal immune tole-rance, contributing to avoid fetal rejection and obste-trical complications. In addition, HLA influences different stages of fetal development, such as embryonic cleavage, trophoblast, formation and implantation. For this review, were surveyed in the MEDLINE and LILACS databases, using the following keywords: "HLA antigens", "pregnancy", "embryonic development", "pregnancy complication", "abortion, habitual", "pre-eclampsia". Knowledge of the HLA role in pregnancy is necessary to improve pregnancy management and autoimmune obstetrical illnesses, by allowing early interventions and specific therapeutics to reduce maternal and perinatal morbidity and mortality.
\end{abstract}

Key words Major histocompatibility complex, HLA antigens, Pregnancy, Immunology, Pregnancy maintenance, Embryonic development, Pregnancy complication, Abortion habitual, Preeclampsia

\section{Resumo}

Este trabalho tem por objetivo discutir a estrutura e função dos Antígenos Leucocitários Humanos (HLA), seus métodos de detecção, nomenclatura e os mecanismos imunopatológicos que o associam com a fisiologia da gestação e morbidades obstétricas. Sabe-se que o equilíbrio imunológico entre mãe e concepto é imprescindível na manutenção da gravidez. Moléculas do HLA - notadamente o HLA-G expresso na interface maternofetal - exercem função importante na tolerância imunológica materna, evitando rejeição fetal e algumas complicações obstétricas. Além disso, o HLA permeia diversas etapas do desenvolvimento conceptual como clivagem, formação do trofoblasto e implantação. Para revisão, foram pesquisados os bancos de dados MEDLINE e LILACS, utilizando os descritores: "HLA antigens"; "pregnancy"; "embryonic development"; "pregnancy complication"; "abortion, habitual"; "preeclampsia". O conhecimento sobre a influência do HLA na gravidez é necessário para melhor manejo da gestação e patologias obstétricas auto-imunes, favorecendo intervenções precoces e terapêutica específica, reduzindo a morbimortalidade materna e perinatal.

Palavras-chave Complexo principal de histocompatibilidade, Antígenos HLA, Gravidez, Imunologia, Conservação da gravidez, Desenvolvimento embrionário, Complicações da gravidez, Aborto habitual, Préeclâmpsia 


\section{Introdução}

O complexo principal de histocompatibilidade (MHC - Major Histocompatibility Complex) é um grande complexo gênico com múltiplos loci cujas moléculas apresentam antígenos protéicos às células do sistema imune, participando assim do processo de rejeição de tecidos estranhos. ${ }^{1-3}$ Em humanos, esse conjunto gênico denomina-se HLA (Human Leukocyte Antigens). O sistema HLA se localiza no braço curto do cromossomo seis humano, sendo herdado em bloco e expresso em co-dominância. ${ }^{2}$ Os loci gênicos do HLA são agrupados em três classes; I, II e III, de acordo com sua estrutura, função e localização. ${ }^{1}$ Os genes $H L A$ de classe I, altamente polimórficos, são responsáveis pela codificação das moléculas de histocompatibilidade clássicas (por exemplo., HLA-A, HLA-B, HLA-C) e não-clássicas (por exemplo, HLA-E, HLA-F, HLA-G). As moléculas clássicas estão presentes em todas as células nucleadas, o HLA-E e HLA-F são expressos em tecidos fetais e o HLA-G predomina na interface materno-fetal, especialmente em citotrofoblastos invasivos, estando potencialmente envolvido na tolerância à gravidez. ${ }^{1,2}$ Os genes de classe II possuem organização mais complexa e são divididos em sub-regiões: $-D R,-D P$ e $-D Q$ e codificam moléculas expressas em células apresentadoras de antígenos como linfócitos B, macrófagos, células dendríticas e alguns tipos de linfócitos T. Os alelos $H L A$ de classe III codificam proteínas importantes para o aparelho imune, como fator de necrose tumoral e componentes do sistema complemento, entre outros. $^{2}$

Moléculas de classe I clássicas funcionam apresentando antígenos oriundos de síntese endógena (via citosólica) para as células T citotóxicas (CD8+). As de classe II apresentam antígenos exógenos às células T auxiliares (CD4+). Genes HLA não-clássicos geralmente codificam proteínas que auxiliam no processamento e transporte antigênico, além de atuarem na imunoregulação de seus sítios específicos. ${ }^{1,3}$

Devido a seu papel central na regulação e resposta imune, o complexo HLA é o único sistema genético convincentemente ligado à susceptibilidade a diversas doenças, servindo modernamente como marcador de risco através da determinação de seus alelos e haplótipos num indivíduo ou população. A hipótese mais aceita para a associação do HLA com doenças é que certas moléculas desse complexo apresentariam peptídeos que levariam à resposta imune deletéria por parte de linfócitos $\mathrm{T}$ específicos, ou em certos casos, por contribuir para o escape imune de moléculas patogênicas. ${ }^{1-3}$
O objetivo dessa revisão é relatar a associação do sistema HLA com alterações imunes da gravidez e algumas complicações obstétricas, mostrando a sua importância como fator de risco e proteção para doenças e como mediador da patogênese desses distúrbios.

\section{Métodos}

Revisão da literatura científica por meio de pesquisa bibliográfica em banco de dados do MEDLINE (PubMed: Cumulative Index Medicus) e LILACS (Literatura Latino-Americana e do Caribe em Ciências da Saúde). Os critérios de seleção incluíam artigos publicados entre 1985 e 2007, escritos em inglês, português ou espanhol, com pesquisas realizadas em seres humanos. Os seguintes termos de pesquisa e operadores booleanos foram utilizados: "HLA Antigens, DNA probes"; "Major Histocompatibility Complex"; "Pregnancy"; "Pregnancy/ immunology"; $\quad$ "Pregnancy/complication"; "Pregnancy Outcome"; "Embryonic Development"; "Abortion, Habitual"; "Pre-eclampsia".

\section{Métodos de detecção do polimorfismo HLA}

Inicialmente, a tipificação do HLA só permitia a identificação dos seus antígenos, pois era realizada por métodos celulares ou sorológicos: microlinfotoxicidade de Terasaki e cultura mista de linfócitos. $\mathrm{O}$ primeiro método verifica reação de citotoxicidade mediada por anticorpo e dependente do complemento e, o segundo, utiliza células com fenótipo conhecido para definir os antígenos HLA. ${ }^{2}$ Avanços recentes na genética molecular disponibilizaram tipificação mais precisa e detalhada, com identificação dos alelos, utilizando o DNA amplificado pela reação em cadeia da polimerase ( $P C R$ - Polimerase Chain Reaction). O SSP (Sequence-specific Primers) e o SSOP (Sequence-specific Oligonucleotides Probes) são os métodos moleculares mais utilizados. ${ }^{1,2}$

\section{Nomenclatura do sistema HLA}

A nomenclatura do sistema HLA sofre evolução e modificações periódicas acompanhando o surgimento de novas técnicas de detecção e identificação destes genes. Um Comitê Internacional é responsável pela nomeação de genes descobertos e modificações na nomenclatura existente., ${ }^{2,3}$

Os testes sorológicos detectam apenas antígenos, os quais podem representar vários alelos. Por esse método, a designação dos loci dos genes HLA é rea- 
lizada pela descrição do prefixo HLA seguida por duas letras maiúsculas, a primeira para identificar a classe (por exemplo, HLA-A, HLA-B, HLA-D) e a segunda para identificar a família (por exemplo, HLA-DR, HLA-DQ). Os antígenos específicos são designados por algarismos arábicos após a identificação do locus (por exemplo, HLA-A1, HLADR3). ${ }^{2}$ Para o locus HLA-C se acrescenta a letra "w" antes do dígito (por exemplo, HLA-Cw1) para evitar confusão com os componentes do sistema complemento. $\mathrm{O}$ "w" também era utilizado para identificar um locus cuja nomenclatura ainda era provisória. ${ }^{2,3}$

Com o uso dos métodos moleculares, iniciados na década de 90, a nomenclatura foi novamente revista. Tal mudança objetivava abranger o polimorfismo descoberto e permitir acrescentar aqueles a serem definidos. Os loci HLA de classe I são descritos pelo prefixo HLA seguidos por uma letra maiúscula para identificar a classe (por exemplo, $H L A-A, H L A-B)$, um asterisco identificando o método como sendo de Biologia Molecular (por exemplo, $H L A-B^{*}$ ) e quatro algarismos (por exemplo, $\left.H L A-B^{*} 0101\right) .{ }^{3}$ Os dois primeiros dígitos correspondem à classificação antigênica (sorológica) e os dois últimos ao alelo específico. ${ }^{2}$ A designação dos loci HLA classe II difere da anterior pelo acréscimo de uma terceira letra maiúscula "A" ou "B" designando as cadeias polimórficas alfa e beta (por exemplo, HLA-DQA1*0101, HLA$D R B 1^{* 0101) . ~ E m ~ 2002, ~ u m a ~ n o v a ~ a t u a l i z a c ̧ a ̃ o ~ d a ~}$ nomenclatura instituiu o acréscimo de mais quatro algarismos, sendo o quinto e o sexto dígitos representantes de variações sinônimas e o sétimo e oitavo, variações nos introns $5^{\prime}$ ou $3^{\prime}$ do gene. ${ }^{3}$

\section{HLA e fisiologia da gravidez}

A relação imunológica entre mãe e feto é importante para a manutenção saudável da gestação, uma vez que o feto possui antígenos de herança paterna que são estranhos para a mãe. Pode-se dizer que o feto é um enxerto semi-alogênico, o qual normalmente não é rejeitado. ${ }^{4}$ Ainda não se tem uma compreensão integral dos mecanismos que protegem o feto do sistema imune materno, mas supõe-se que o trofoblasto e a expressão do $H L A-G$ não-clássico exerçam função importante nesta tolerância imunológica, uma vez que representam a interface entre as células fetais e maternas. ${ }^{5}$ Caso as células trofoblásticas fetais expressem moléculas HLA paternas, o sistema imune materno poderá reconhecê-las como estranhas, desencadeando rejeição ao concepto. Normalmente isso não ocorre porque as principais moléculas do HLA responsáveis pela rejeição, HLA-A, -B e HLA de classe II, não estão presentes nas células do trofoblasto. Apenas moléculas não-clássicas do HLA, com baixo polimorfismo, como HLA-G, -E, -F e algumas moléculas HLA-C, são expressas. ${ }^{6}$

O HLA-G tem sido descrito como mediador da tolerância materno-fetal, pois está expresso nas células citotrofoblásticas extravilosas invasivas, células do endotélio endovascular fetal e células do tecido e líquido amniótico que entram em contato com o sistema imune materno. A influência do HLA$\mathrm{G}$ na evolução da gestação não é totalmente elucidada, mas tem sido sugerido seu envolvimento na diferenciação do tecido fetal, implantação do blastocisto, angiogênese placentária e principalmente na prevenção do ataque ao trofoblasto inibindo ação das células NK (Natural Killer) e T citotóxicas. ${ }^{5,6} \mathrm{O}$ $H L A-G$ pode ser expresso através de cinco isoformas: três isoformas de $H L A-G$ transmembrana $(-G 1,-G 2,-G 3)$ e duas isoformas solúveis circulantes (-G5 e-G6). É possível que estas últimas atuem como imunossupressores na gravidez, principalmente inibindo a expressão de CD8, indispensável na ativação da citotoxicidade mediada por células. Porém, o baixo polimorfismo do $H L A-G$ por si só pode ser indutor da tolerância materna. Secreção de HLA-G solúvel pelos embriões parecem ser necessários à implantação e clivagem, orientando o sucesso de fertilizações in vitro, favorecendo a seleção de embriões mais aptos. O papel dessas moléculas sugere que o sucesso da gravidez suplanta as funções do trofoblasto e envolvem complexos mecanismos imunomoleculares e de citocinas. 5,6

Sabe-se atualmente que homozigose na deleção do HLA-G1 pode não expressar repercussões deletérias à gestação, já que as outras isoformas do $H L A-G$, particularmente - $G 2$, podem desencadear estímulos alternativos à imunotolerância. Além disso, ainda é incerto o papel dos genes HLA-E e - F neste contexto, com alguns estudos sugerindo compartilhamento de funções ou até mesmo sinergismo entre elas e o $H L A-G{ }^{4}$ Tais mecanismos constituem defesa evolutiva importante à manutenção da espécie e parecem ter sido selecionados durante as gerações. ${ }^{4}$

\section{HLA e complicações obstétricas}

\section{Aborto espontâneo recorrente}

O Aborto Espontâneo Recorrente (AER) é uma síndrome caracterizada por três ou mais episódios de aborto consecutivos e espontâneos. ${ }^{7,8}$ As alterações cromossômicas fetais são responsáveis pela maior 
parte das perdas. Distúrbios hormonais e anormalidades anatômicas uterinas e endometriais também são causas freqüentes. Contudo, cerca de $50 \%$ não possuem etiologia conhecida. Em tais casos, os distúrbios do sistema imune são considerados como prováveis causadores de AER inexplicável. ${ }^{8,9}$ Uma hipótese é que a presença de HLA em homozigose no enxerto embrionário (semelhança do par de genes HLA entre os pais) resultaria numa produção diminuída de anticorpos "bloqueadores" maternos. Tais anticorpos seriam responsáveis pela proteção do feto contra a resposta imune da mãe, ocasionando a tolerância fetal, suprimindo a rejeição e evitando o aborto. ${ }^{7}$ Estudos iniciais associavam o compartilhamento de antígenos HLA classe I pelos pais com a predisposição ao AER. ${ }^{7}$

Mais recentemente, as alterações na expressão trofoblástica do HLA de classe I, particularmente o $G$ e $-E$, são sugeridas como causadores do AER. Certos alelos polimórficos associados ao $H L A-G$ que interferem na gênese das formas transmembrana (G1) e solúveis (G5) dessa molécula, particularmente os alelos HLA-G*0104, $-G^{*} 0105 N e$ $G^{*} 01013$, se associam à expressão defeituosa ou diminuída destas, além de exercer interferência negativa na expressão e ação do $H L A-E .^{8}$ Tais fatores resultariam na quebra da tolerância materna ao feto e consequiente aborto. ${ }^{8}$ Contudo, a literatura apresenta dados divergentes em relação a essa hipótese e o papel exato dos mecanismos descritos necessitam de mais estudos comprobatórios. ${ }^{9}$ A presença de certos alelos HLA classe II parecem interferir na predisposição a esse distúrbio. Wang et $a l .{ }^{10}$ encontraram maior susceptibilidade associada à presença de $H L A-D Q B 1$ e com os haplótipos $H L A$ $D Q B 1 * 0604-D Q B 1 * 0605$, enquanto que as portadoras dos alelos $H L A-D Q B 1 * 0501$ e $-D Q B 1 * 0502$ pareciam ser protegidas. ${ }^{10}$ Kruse et al. ${ }^{7}$ observaram que o alelo $H L A-D R B 1 * 03$ conferia predisposição. Além disso, esse alelo é associado à produção de anticorpos anti-cardiolipina e anticorpos antinucleares que estão presentes em muitas mulheres com aborto espontâneo recorrente, podendo ser possíveis agentes influenciadores da fisiopatologia desse distúrbio. ${ }^{7}$

\section{Pré-eclâmpsia}

Trata-se de complicação comum e grave da gravidez, mais freqüente em primogestações. Fatores imunogenéticos, especialmente o HLA, são aspectos centrais nas suposições etiopatogênicas desta síndrome, atuando tanto na predisposição, com componente familial importante, quanto no desen- cadeamento do quadro patológico. ${ }^{11}$ A pré-eclâmpsia (PE) resulta de má formação placentária, tornando-a ineficiente na perfusão e proteção do feto à defesa imune materna. A baixa expressão de $H L A-G$ em trofoblastos placentários e no soro de mulheres com pré-eclâmpsia é freqüiente, e se relacionam a alterações vasculares placentárias, prematuridade e baixo peso fetal. Placentas isquêmicas são responsáveis pela PE e sua evolução. Um estudo recente demonstrou que, gestantes com queda importante nos níveis séricos de HLAG1/G5 solúveis, no segundo trimestre eram mais propensas a desenvolver préeclampsia, sugerindo tal medida como marcador prénatal dessa complicação. ${ }^{12}$ Além disso, presença de determinados antígenos HLA é comum em mulheres acometidas. Antígenos HLA-DR2, -DR4, -DR5, DRw8, -DRw10, -DRw11, -DRw18 e DQw2 e o alelo $-D Q B 1 * 04$ são associados a maior risco e o - $Q w 3$ parece ser protetor. A freqüência dos haplótipos HLA-DR4-DRw11-DQw2 (envolvido em maior atuação linfocitária e de citocinas inflamatórias) e -B44-DR7 é maior em grávidas com PE. ${ }^{11,13}$ Moléculas HLA-DR53 se associam com a síndrome do anticorpo antifosfolípide, por si só grande fator de risco para PE. ${ }^{13}$ É possível ainda que a PE seja um distúrbio do casal, pois a homozigose para genes $H L A-D R$ se relaciona a maior risco para a síndrome. ${ }^{14}$

\section{Doenças auto-imunes e gravidez}

Há poucos anos, a presença de doença auto-imune era contra-indicação absoluta à gravidez. Os avanços no tratamento e acompanhamento destas pacientes tornaram possível a gestação e o controle da morbimortalidade materno-fetal, além de viabilizar estudos imunogenéticos nestas condições. Sabe-se que a expressão de $H L A-G$, abundante na interface materno-fetal, altera o balanço entre resposta imune Th1/Th2, inibindo reações celulares de defesa e resultando, de uma maneira simplista, no estado "imunodeprimido fisiológico" da grávida, necessário para tolerância imune ao feto. Tal condição a torna mais susceptível a infecções, mas parece atenuar as manifestações das patologias auto-imunes. Moléculas HLA e mecanismos de mimetismo molecular parecem participar da fisiopatologia. ${ }^{15}$ Diferenças nos antígenos HLA de classe II entre mãe e feto são associados à remissão ou melhora clínica de algumas patologias auto-imunes como artrite reumatóide, particularmente o HLA-DQA1. Observa-se ainda maior incidência de pênfigo gestacional e esclerose múltipla associado à família alélica $H L A-D R B 1 * 01$ até $-D R B 1 * 14$ em gestantes 
de fetos HLA homozigóticos. ${ }^{15,16}$ Os mecanismos exatos dessa relação não são bem esclarecidos, mas é provável que o fluxo de células entre mãe e feto HLA compartilhado (homozigótico) seja favorecido e que tais células no organismo materno desencadeiam resposta imune com produção de anticorpos, pois não são completamente compatíveis. No entanto, devido à grande semelhança das células compartilhadas, tais anticorpos podem atacar estruturas próprias da mãe (funcionando como auto-anticorpos) e participar da gênese, manutenção ou piora das doenças auto-imunes na gravidez. ${ }^{16}$

\section{Hipertensão induzida pela gravidez}

A hipertensão induzida pela gravidez (HIG) é importante fator de morbimortalidade para a gestante e seu concepto. A etiologia desse distúrbio é pouco conhecida, mas é possível que más adaptações imunes interfiram na patogênese. ${ }^{17}$ Uma explicação é que a exposição do feto aos antígenos HLA-DR maternos suscite resposta imune com a produção de anticorpos fetais contra aquele HLA. Em contrapartida, a mãe desenvolve resposta humoral aos anticorpos fetais. Tal mecanismo é aventado como predisponente a estados hipertensivos em estudos bem controlados. ${ }^{17,18}$ Uma maior prevalência dessa condição é observada em mulheres que compartilham antígenos HLA com seus esposos ou fetos. O alelo $H L A-D Q A 1$ compartilhado entre casais foi associado a HIG em um estudo chinês. Nesse mesmo estudo, o alelo $H L A-D Q B 1 * 0502$ foi marcador de susceptibilidade para HIG. ${ }^{19}$

\section{Diabetes e intolerância a glicose gestacionais}

A investigação de potenciais fatores predisponentes ao Diabetes Melito Gestacional (DMG) e à Intolerância à Glicose durante a Gravidez (IGG), tais como as moléculas do HLA, é de suma importância tendo em vista as complicações obstétricas oriundas dessas condições, além do risco da permanência desses distúrbios em quase $50 \%$ das mulheres acometidas no pós-parto. ${ }^{20,21}$

A patogênese da DMG não é bem esclarecida. Supõe-se que diminuição na imunotolerância materna ao concepto leve à formação de anticorpos anti-HLA classe II e conseqüente resposta inflamatória e humoral sistêmica materna, predispondo ao DMG. $^{21}$ A presença de anticorpos anti-HLA classe II foram significativamente positivos em gestantes com DMG comparado a controles sadios. ${ }^{21}$ Além disso, a presença de antígenos fetais na circulação materna poderia atuar na manutenção da DMG durante a gestação. A persistência dessas moléculas após a gravidez explicaria os diabetes persistentes no período pós-parto. ${ }^{21}$

Em um estudo realizado na França, Vambergue et al., ${ }^{20}$ não observaram diferenças significativas entre controles sadios, grávidas tolerantes à glicose ou diabéticas, quanto à freqüência de alelos $H L A$ $D R B 1 * 03$ e $-D R B 1 * 04$, conhecidos marcadores de susceptibilidade em diabetes melito não gestacional. No entanto, mulheres com DMG portadoras de anticorpos anti-ilhotas pancreáticas, ou que desenvolvem diabetes insulino-dependente pós-parto, possuem antígenos HLA típicos da predisposição ao diabetes melito tipo 1 como, por exemplo, DR3 e DR4, sugerindo que a gravidez pode funcionar como um "gatilho" para o desenvolvimento do diabetes em mulheres predispostas. ${ }^{21}$ Outro estudo recente observou que o diabetes auto-imune e não autoimune iniciados durante a gravidez se relacionam com genótipos HLA distintos. ${ }^{22}$ Haplótipos $H L A$ $D R 3-D Q 2$ e-DR4-DQ8 foram mais frequientes em DMG auto-imune, conferindo maior risco para suas portadoras. Haplótipos $H L A-D R 7-D Q 2$, - DR9-DQ9, -DR14-DQ5 foram associados a maior predisposição para o DMG não auto-imune.

Em mulheres francesas, haplótipos HLA$D R B 1 * 0101-D Q A 1 * 0101-D Q B 1 * 0501$ e $D R B 1 * 1302-D Q A 1 * 0102-D Q B 1 * 0604$ são associados a IGG. Já o haplótipo HLA-DRB1*0701$D Q A 1 * 0201-D Q B 1 * 02$ tem associação positiva com o DMG, e o -DRB1*1103-*1104 foi visto em menor freqüência nesse grupo. ${ }^{20} \mathrm{Em}$ mulheres japonesas, alelos $H L A-D R 6$ se associaram com a susceptibilidade ao $D M G$ e HLA-DR2 e -DR51 à proteção. As presenças do alelo $H L A-D R 3$ e haplótipo -DR6-DR9 se associou à doença com pior prognóstico. ${ }^{23}$ Tais dados mostram que o padrão de susceptibilidade varia como grupo racial estudado, indicando necessidade de estudos com população local para seu uso e aplicações.

\section{Neoplasias trofoblásticas gestacionais}

Genes do HLA atuam no desenvolvimento e diferenciação das Neoplasias Trofoblásticas Gestacionais (NTG) bem como na predisposição individual a desenvolvê-las. A comparação da expressão de $H L A$ $D R B 1$ e $-D Q B 1$ entre a decídua e a neoplasia trofoblástica pode ajudar na diferenciação entre molas parciais e completas, principalmente quando a histologia é incerta. ${ }^{24}$ A capacidade de expressar HLA-G, molécula associada ao escape da defesa imune materna, e HLA-E, que auxilia na resistência à ação das células NK, determina o caráter de 
tolerância imune e invasidade observadas nos casos de coriocarcinomas. ${ }^{25}$ Antígenos HLA-DR6 são freqüentes em mulheres com NTG, e alelos do $H L A$ $D R$ compartilhados pelo casal sinalizam maior predisposição a esse evento. Haplótipos HLA-Bw35DR5 também são associados positivamente à $\mathrm{NTG}^{26}$ Mola hidatiforme é mais freqüente em portadoras de antígenos HLA-A1, e compatibilidade parcial de locus $H L A-B$ com o parceiro foi associada a maior risco de desenvolver essa neoplasia. ${ }^{27}$

\section{Vírus da imunodeficiência humana e gravidez}

O complexo HLA pode marcar padrões de susceptibilidade ou proteção à transmissão vertical e a infecção perinatal do Vírus da Imunodeficiência Humana (HIV). Mães portadoras de um conjunto de genes funcionantes relacionados ao HLA classe I, o supertipo A2/6802, possuem menor susceptibilidade à infecção pelo HIV e têm menores taxas de transmissão vertical do vírus. ${ }^{28}$ Hipóteses recentes sugerem influência das variantes do $H L A-G$ envolvidos nesse processo. A similaridade entre seqüências do DNA do HLA-G em mãe e concepto foi associada a maior risco de transmissão vertical do HIV, pois isso impediria o feto de reconhecer as células maternas infectadas como estranhas e desencadear resposta imune seletiva protetora. Alelos classe II, $H L A-B * 1302,-B * 3501,-B * 3503,-B * 4402$, $-B * 5001$ se associam a maior probabilidade de infecção vertical, enquanto $-B * 4901 e-B * 5301$ conferem proteção através de interação imune com células NK placentárias. ${ }^{29}$

O conjunto HLA do feto também atua nestas situações. O alelo $H L A-B * 18$ foi associado a baixo risco de transmissão precoce (infecção detectada no bebê até o primeiro mês) ou tardia (detectada após o primeiro mês e atribuída à amamentação), sugerindo ser este alelo um importante fator no estudo de uma vacina contra o HIV. ${ }^{30}$ Lactentes portadores do alelo $H L A A^{* 29}$ foram mais susceptíveis à infecção precoce, já o $H L A-C w^{*} 07 e-C w^{*} 08$ se associaram à forma tardia. ${ }^{30}$

\section{Conclusões}

O complexo HLA e seus fatores regulatórios atuam em quase todas as etapas do desenvolvimento embrionário e são fundamentais no sucesso reprodutivo. Tipos específicos, bem como aumento ou diminuição da expressão de moléculas HLA marcam o desenvolvimento dos gametas, a clivagem embrionária, a formação do trofoblasto e do blastocisto, a implantação, desenvolvimento e sobrevivência fetal. Desequilíbrios nesta interação imune participam da fisiopatologia da maioria dos processos patológicos obstétricos, levando a riscos potencialmente fatais para a mãe e o concepto, ônus socialmente grave e temível. Mais estudos sobre a influência e valor preditivo do complexo HLA nestes aspectos, especialmente em população local, poderia dirigir melhores técnicas de manejo destas pacientes, favorecendo intervenções precoces e terapêuticaespecífica, diminuindo a morbimortalidade materna e perinatal.

\section{Referências}

1. Klein J, Sato A. The HLA System. First of two parts. New Engl J Med. 2000; 343: 702-9.

2. Alves C, Veiga S, Souza T, Toralles MB, Silva-Bacellar AL. Papel del sistema de histocompatibilidad humano en la patogénesis de las enfermedades neurológicas. Rev Neurol. 2007; 44: 298-302.

3. Marsh SG, Albert ED, Bodmer WF, Bontrop RE, Dupont B, Erlich HA, Geraghty DE, Hansen JA, Mach B, Mayr WR, Parham P, Petersdorf EW, Sasazuki T, Schreuder GM, Strominger JL, Svejgaard A, Terasaki PI. Nomenclature for factors of the HLA system, 2002. Tissue Antigens. 2002; 60: 407-64.

4. Hunt JS. Stranger in a strange land. Immunol Rev. 2006; 213: $36-47$.
5. Hviid TV. HLA-G in human reproduction: aspects of genetics, function and pregnancy complications. Hum Reprod Updat. 2006; 12: 209-32.

6. Moscoso J, Serrano-Vela JI, Pacheco R, Arnaiz-Villena A. HLA-G, -E and -F: allelism, function and evolution. Transpl Immunol. 2006; 17: 61-4.

7. Kruse C, Steffensen R, Varming K, Christiansen OB. A study of HLA-DR and -DQ alleles in 588 patients and 562 controls confirms that HLA-DRB $1 * 03$ is associated with recurrent miscarriage. Hum Reprod. 2004; 19: 1215-21.

8. Ober C, Billstrand C, Kuldanek S, Tan Z.The miscarriageassociated HLA-G -725G allele influences transcription rates in JEG-3 cells. Hum Reprod. 2006; 21: 1743-8. 
9. Bhalla A, Stone PR, Liddell HS, Zanderigo A, Chamley LW. Comparison of the expression of human leukocyte antigen (HLA)-G and HLA-E in women with normal pregnancy and those with recurrent miscarriage. Reproduction. 2006; 131: 583-9.

10. Wang Xi-peng, LIN Qi-de, Lu Pei-hua, MA Zheng-wen, ZHAO Ai-min. Association of HLA-DQB1 coding region with unexplained recurrent spontaneous abortion. Chin Med J. 2004; 117: 492-7.

11. Sargent IL, Borzychowski AM, Redman CW Immunoregulation in normal pregnancy and pre-eclampsia: an overview. Reprod Biomed Online. 2006; 13: 680-6.

12. Steinborn A, Varkonyi T, Scharf A, Bahlmann F, Klee A, Sohn C. Early Detection of Decreased Soluble HLA-G Levels in the Maternal Circulation Predicts the Occurrence of Preeclampsia and Intrauterine Growth Retardation During Further Course of Pregnancy. Am J Reprod Immunol. 2007; 57: 277-86.

13. Honda K, Takakuwa K, Hataya I, Yasuda M, Kurabayashi T, Tanaka K. HLA-DQB1 and HLA-DPB1 genotypes in severe preeclampsia. Obstet Gynecol. 2000; 96: 385-9.

14. Brunori IL, Battini L, Simonelli M, Brunori E, Valentino V, Curcio M, Mariotti ML, Lapi S, Genazzani AR. HLA-DR in couples associated with preeclampsia: background and updating by DNA sequencing. J Reprod Immunol. 2003; 59: 235-43.

15. Gordon C. Pregnancy and autoimmune diseases. Best Pract Res Clin Rheumatol. 2004; 18: 359-79.

16. Nelson JL. HLA relationships of pregnancy, microchimerism and autoimmune disease. J Reprod Immunol. 2001; 52: 77-84.

17. Mahmoud F, Omu A, Abul H, El-Rayes S, Haines D. Lymphocyte subpopulations in pregnancy complicated by hypertension. J Obstet Gynaecol. 2003; 23: 20-6.

18. Hoff C, Peevy K, Giattina K, Spinnato JA, Peterson RD.Maternal-fetal HLA-DR relationships and pregnancyinduced hypertension. Obstet Gynecol. 1992; 80:1007-12.

19. Mao Y, Zhang Z, Fan L, Wu Q, Jiang L, Yang J, Yao F.HLA-DQA1, -DQB1 polymorphism distribution in Chinese women with pregnancy induced hypertension in Shanghai area. Chin Med J. 1998; 111:163-5.

20. Vambergue A, Fajardy I, Bianchi F, Cazaubiel M, VerierMine O, Goeusse P, Fontaine P, Danze PM. Gestational diabetes mellitus and HLA class II (-DQ, -DR) association: The Digest Study. Eur J Immunogenet. 1997; 24: 385-94.
21. Steinborn A, Saran G, Schneider A, Fersis N, Sohn C, Schmitt E. The presence of gestational diabetes is associated with increased detection of anti-HLA-class II antibodies in the maternal circulation. Am J Reprod Immunol. 2006; 56: $124-34$

22. Torn C, Gupta M, Sanjeevi CB, Aberg A, Frid A, LandinOlsson M. Different HLA-DR-DQ and MHC class I chainrelated gene A (MICA) genotypes in autoimmune and nonautoimmune gestational diabetes in a Swedish population. Hum Immunol. 2004; 65: 1443-50.

23. Song D, Liu Y, Han Y, Shang G, Hua S, Zhang H, Guo S, Jiao S. Study on the gestational diabetes mellitus and histocompatibility human leukocyte antigen DRB allele polymorphism. Zhonghua Fu Chan Ke Za Zhi. 2002; 37: 284-6.

24. Bateman AC, Hemmatpour SK, Theaker JM, Howell WM. Genetic analysis of hydatidiform moles in paraffin wax embedded tissue using rapid, sequence specific PCR-based HLA class II typing. J Clin Pathol. 1997; 50: 288-93.

25. Sivori S, Parolini S, Marcenaro E, Millo R, Bottino C, Moretta A.Triggering receptors involved in natural killer cell-mediated cytotoxicity against choriocarcinoma cell lines. Hum Immunol. 2000; 61:1055-8.

26. Sbracia M, Scarpellini F, Mastrone M, Grasso JA. HLA antigen sharing in Italian couples in which women were affected by gestational trophoblastic tumors. Am J Reprod Immunol. 1996; 35: 252-5.

27. Souka AR, Kholeif A, Zaki S, Rocca M, G hanem I. Human leukocyte antigen in hydatidiform mole. Int $\mathrm{J}$ Gynaecol Obstet. 1993; 41: 257-60.

28. Aikhionbare FO, Kumaresan K, Shamsa F, Bond VC. HLAG DNA sequence variants and risk of perinatal HIV-1 transmission. AIDS Res Ther. 2006; 23: 28-36.

29. Winchester R, Pitt J, Charurat M, Magder LS, Goring HH, Landay A, Read JS, Shearer W, Handelsman E, Luzuriaga K, Hillyer GV, Blattner W. Mother-to-child transmission of HIV-1: strong association with certain maternal HLA-B alleles independent of viral load implicates innate immune mechanisms. J Acquir Immune Defic Syndr. 2004; 36(2): 659-70.

30. Farquhar C, Rowland-Jones S, Mbori-Ngacha D, Redman M, Lohman B, Slyker J, Otieno P, Obimbo E, Rostron T, Ochieng J, Oyugi J, Bosire R, John-Stewart G. Human leukocyte antigen (HLA) $\mathrm{B} * 18$ and protection against mother-to-child HIV type 1 transmission. AIDS Res Hum Retroviruses. 2004; 20: 692-7. 